\title{
Determination of the limiting angle of inclination of tubular belt conveyor
}

\author{
Ruslan Kiriia ${ }^{1, *}$, Andrii Smirnov ${ }^{1}$, Tetiana Zhyhula $^{1}$, and Todor Zhelyazov ${ }^{2}$ \\ ${ }^{1}$ Institute of Geotechnical Mechanics named by N. Poljakov of National Academy of Sciences of \\ Ukraine, 49005, Dnipro, Simferopolska Str., 2a, Ukraine \\ ${ }^{2}$ Technical University of Sofia, 1000, Sofia, Kliment Ohridski Blvd, 8, Bulgaria
}

\begin{abstract}
Currently, the widespread use of tubular belt conveyors is constrained by insufficient justification of the conveyor parameters. In the work, on the basis of the theory of the limiting equilibrium state of bulk load, equations of equilibrium of load on the belt of an inclined tubular conveyor are obtained. It is assumed that the belt was a rigid cylinder filled with bulk material in extreme equilibrium. Based on the obtained equilibrium equation, the analytical dependences of the limiting angle of inclination of the belt conveyor with a tubular belt on the degree of unfilling with the bulk load of the belt and the properties of the bulk load are found. From the analysis of these dependencies it follows that the limiting angle of inclination of the tubular belt conveyor depends on the angle of internal friction of the transported load, the angle of friction of the load on the conveyor belt, the angle of unfilling of the belt with load and does not depend on the radius of the conveyor belt.
\end{abstract}

\section{Introduction}

At present, belt conveyors with tubular belt are widely used. The main advantage of the tubular belt conveyor is the lack of contact of the load with the environment, as well as the ability to transport load along curved routes without reloading nodes.

In addition, unlike a conveyor belt with a deep grooved belt, the transported load on the conveyor belt is more stable due to the limited space.

Perten Yu.A., Chernenko V.D., Galkin V.I., Dmitriev V.G. are engaged in questions of stability and the maximum angle of inclination of the tubular belt conveyor.

In [1], the problem of stability of bulk load of an inclined tubular conveyor is solved, while the load when moving along the tubular conveyor belt is affected by the friction forces of the load on the belt, directed along the belt, components of the load's gravity directed against the movement belts, and the bulk load back up force, directed in the same way as the friction force, along the movement of the belt.

However, such a mode of equilibrium of bulk load in a tubular conveyor is possible with conveyor tilt angles much higher than permissible.

In [2], on the basis of theories about anisotropic thin shells and the limiting equilibrium state of granular media, the stability problem of bulk load on inclined and curved sections

\footnotetext{
${ }^{*}$ Corresponding author: kiriya.igtm@gmail.com
} 
of a tubular belt conveyor was solved. At the same time, as in [1], it was assumed that in addition to the friction forces of the load on the belt and the component of the gravity of the load, the load back up force along the movement of the conveyor belt was also taken into account. At the same time, the bulk load was small-sized.

In [3], the results of experimental studies on the determination of the permissible inclination angles of conveyors with a deep groove belt and daisy-colored roller supports are considered. It is found that the permissible angle of inclination of the conveyor depends on the degree of filling of the conveyor belt with the load, the angle of internal friction of the load, particle size distribution and humidity of the load and practically does not depend on the speed of the conveyor belt.

From the analysis of these works it follows that the question of determining the maximum angle of inclination of the tubular belt conveyor transporting large fraction bulk loads has not been sufficiently studied.

The purpose of this article is to establish, on the basis of the theory of the limit state of bulk load, the dependence of the limiting angle of inclination of a tubular belt conveyor on the parameters of the conveyor, the properties of the belt and the load.

\section{Methods}

Suppose that the belt is a rigid cylinder filled with bulk material and inclined to the horizon at an angle $\alpha$ (Fig. 1).
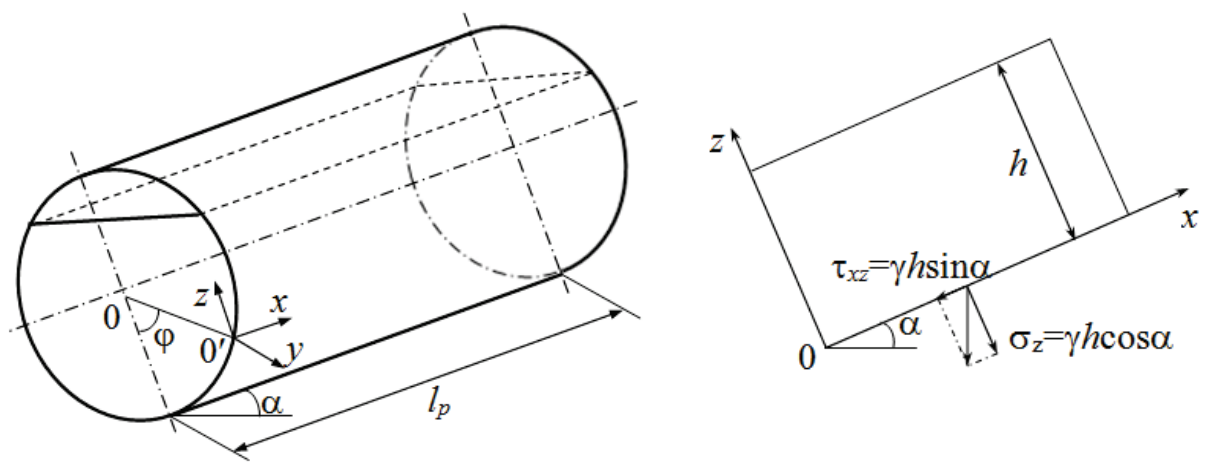

Fig. 1. The stress-strain state of the load inclined conveyor with a tubular belt.

The load is affected by the friction forces of the load on the conveyor belt, directed against the movement of the belt, and the component of the gravity of the load, directed along the movement of the conveyor belt.

Suppose that the coefficient of friction of the load on the belt $f_{1}$ is less than the coefficient of internal friction of the bulk load $f\left(f_{1}<f\right)$. Since otherwise, i.e. at $f_{1} \geq f$, the maximum angle of inclination of the tubular conveyor $\alpha_{n}=\operatorname{arctg} f$.

Let us draw up the equation of balance of forces acting on the bulk load section between normal sections of the belt at the locations of the roller support, i.e. section of bulk load between the roller supports.

In the projection onto the conveyor axis, the equation of balance of forces acting on this section of bulk load will take the form:

$$
F_{T}=F_{g}
$$

where $F_{T}$ is the friction force of the selected load section on the conveyor belt, directed by the movement of the conveyor belt, $\mathrm{N} ; F_{g}$ is a component of the weight force of the selected 
load section, directed against the movement of the conveyor belt, $\mathrm{N}$.

According to [4], active $\sigma_{b a}$ and passive $\sigma_{b n}$ normal lateral pressures and, which depend on the degree (corner) of unfilling of the belt $\theta$ and the angular coordinate $\varphi$ of the points of the conveyor belt, act on the tubular conveyor belt from the load side (Fig. 2).

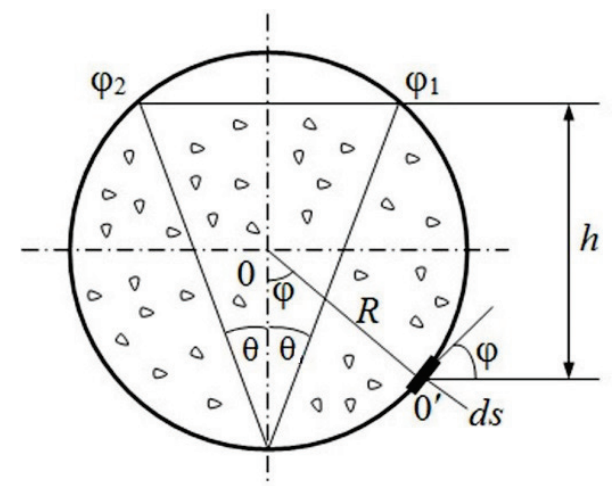

Fig. 2. Cross section of a tubular conveyor belt with load.

In this case, active lateral pressure $\sigma_{b a}$ acts on the belt from the load side to the middle of the span between the roller supports, and passive lateral pressure $\sigma_{b n}$ acts on the sides of the belt from the middle of the span to its end.

The average normal pressure acting on the belt of the tubular conveyor on the load side is determined by the formula:

$$
\sigma_{c}=\frac{\sigma_{b a}+\sigma_{b \Pi}}{2}
$$

where $\sigma_{b a}, \sigma_{b n}$ is active and passive lateral pressures acting on the tubular belt of the conveyor, $\mathrm{N} / \mathrm{m}^{2}$.

The average tangential stresses acting on the belt from the load side and directed along the belt, we define by the formula:

$$
\tau_{c}=f_{1} \sigma_{c}
$$

where $f_{1}$ is the coefficient of friction of the load on the conveyor belt.

Select a small element of the arc of the belt $d s$ at the point of the belt with the angular coordinate $\varphi$ (Fig. 2).

The total friction force of the selected area of bulk load on the conveyor belt is determined by the formula:

$$
F_{T}=1_{p} \int \tau_{c} d s
$$

where $d s$ is the arc element of the normal section of the conveyor belt, equal $d s=R d \varphi, \mathrm{m} ; R$ is the radius of the normal section of the tubular belt, $\mathrm{m} ; l_{p}$ is the distance between the rollers, $\mathrm{m} ; \varphi$ is the angular coordinate of the points of the tubular conveyor belt, rad.

Substituting the expression in the right part under the integral $\tau_{c}$, according to formulas (3) and (2), we obtain:

$$
F_{T}=f_{1} 1_{p} \int_{\varphi_{1}}^{\varphi_{2}} \frac{\sigma_{b a}+\sigma_{b \Pi}}{2} R d \varphi
$$


where $\varphi_{1}$ and $\varphi_{2}$ are the limits of integration $\left(\varphi_{1}=\pi-2 \theta ; \varphi_{2}=-\pi+2 \theta\right) ; \theta$ is the angle of unfilling of the belt, rad. (Fig. 2).

Dividing the region of integration on $\varphi$ on left and right to part, we obtain:

$$
F_{T}=f_{1} 1_{p} R \int_{0}^{\varphi_{1}}\left(\sigma_{b a}+\sigma_{b I}\right) d \varphi
$$

Now we define the normal active and passive bulk pressure acting on the conveyor belt.

According to $[5,6]$, the normal $\sigma_{z}$ and tangential $\tau_{x z}$ stresses acting on the selected element of the belt from the side of bulk load are determined by the formulas (Fig. 1):

$$
\sigma_{z}=\gamma h \cos \alpha ; \quad \tau_{x z}=\gamma h \sin \alpha .
$$

where $h$ is the distance from the surface of the bulk load to the selected element of the belt, $\mathrm{m} ; \gamma$ is the specific gravity of the load, $\mathrm{N} / \mathrm{m}^{3} ; \alpha$ is the angle of the conveyor, deg.

From Figure 2, the distance $h$ is determined by the formula:

$$
h=R(\cos 2 \theta+\cos \varphi) \text {. }
$$

From the Mohr circle $[5,6]$, the smallest and greatest normal stresses in the bulk load $\sigma_{x}$ at the point of contact of the bulk load with the selected element of the belt and perpendicular to the ZY plane are determined from the expression:

$$
\sigma_{x}=\sigma_{z}\left(1+2 f^{2}\right) \mp 2 \sqrt{\left(1+f^{2}\right)\left(\sigma_{z}^{2} f^{2}-\tau_{x z}^{2}\right)} .
$$

Substituting (8) into (9), after the conversion, we obtain the smallest $\sigma_{x a}$ and greatest $\sigma_{x n}$ stresses $\sigma_{x}$ :

$$
\begin{aligned}
& \sigma_{x a}=\gamma h \cos \alpha\left[1+2 f^{2}-2 \sqrt{\left(1+f^{2}\right)\left(f^{2}-\operatorname{tg}^{2} \alpha\right)}\right] \\
& \sigma_{x I}=\gamma h \cos \alpha\left[1+2 f^{2}+2 \sqrt{\left(1+f^{2}\right)\left(f^{2}-\operatorname{tg}^{2} \alpha\right)}\right]
\end{aligned}
$$

In this case, the greatest and smallest normal stresses of bulk load acting on the selected element of the belt, perpendicular to the XY plane, are determined by the formulas [6]:

$$
\sigma_{y a}=\frac{\sigma_{x a}+\sigma_{z}}{2}-r_{1} ; \quad \sigma_{y I}=\frac{\sigma_{x I}+\sigma_{z}}{2}+r_{2}
$$

where $r_{1,2}=f\left(\sigma_{z} \sqrt{1+f^{2}} \mp \sqrt{\sigma_{z}^{2} f^{2}-\tau_{x z}}\right)$.

Substituting (10) into (11) taking into account (7), after the conversion, we obtain:

$$
\sigma_{y a}=\gamma h \cos \alpha \cdot n_{1} ; \quad \sigma_{y I}=\gamma h \cos \alpha \cdot n_{2} .
$$

Here $n_{1}$ and $n_{2}$ are the smallest and largest coefficients of lateral thrust, determined by the formulas:

$$
n_{1}=1+f^{2}-\sqrt{\left(1+f^{2}\right)\left(f^{2}-\operatorname{tg}^{2} \alpha\right)}-f\left(\sqrt{1+f^{2}}-\sqrt{f^{2}-\operatorname{tg}^{2} \alpha}\right)
$$




$$
n_{2}=1+f^{2}+\sqrt{\left(1+f^{2}\right)\left(f^{2}-\operatorname{tg}^{2} \alpha\right)}+f\left(\sqrt{1+f^{2}}+\sqrt{f^{2}-\operatorname{tg}^{2} \alpha}\right)
$$

Substituting $\alpha=0$ in formulas (13), we obtain

$$
n_{1}=1+2 f^{2}-2 f \sqrt{1+f^{2}}=m ; \quad n_{2}=1+2 f^{2}+2 f \sqrt{1+f^{2}}=\frac{1}{m}
$$

where $m$ is the coefficient of mobility of bulk load [6].

Active and passive lateral pressures of bulk load acting on the selected element of the conveyor belt, we define by the formulas:

$$
\begin{aligned}
& \sigma_{b a}=\sigma_{z} \cos ^{2} \varphi+\sigma_{y a} \sin ^{2} \varphi ; \\
& \sigma_{b n}=\sigma_{z} \cos ^{2} \varphi+\sigma_{y n} \sin ^{2} \varphi .
\end{aligned}
$$

Substituting expressions (7) and (12) in (15) and (16), after the transformation we obtain:

$$
\begin{gathered}
\sigma_{b a}=\gamma h \cos \alpha\left(\cos ^{2} \varphi+n_{1} \sin ^{2} \varphi\right) ; \\
\sigma_{b n}=\gamma h \cos \alpha\left(\cos ^{2} \varphi+n_{2} \sin ^{2} \varphi\right) .
\end{gathered}
$$

Or, taking into account (8), we have

$$
\begin{gathered}
\sigma_{b a}=\gamma R \cos \alpha(\cos 2 \theta+\cos \varphi)\left(\cos ^{2} \varphi+n_{1} \sin ^{2} \varphi\right) \\
\sigma_{b n}=\gamma R \cos \alpha(\cos 2 \theta+\cos \varphi)\left(\cos ^{2} \varphi+n_{2} \sin ^{2} \varphi\right)
\end{gathered}
$$

Substituting (19) and (20) in the right side of the expression (6), after the conversion we get:

$$
F_{T}=2 \gamma f_{1} l_{p} R^{2} \cos \alpha \int_{0}^{\varphi_{1}}(\cos 2 \theta+\cos \varphi)\left(\cos ^{2} \varphi+\frac{n_{1}+n_{2}}{2} \sin ^{2} \varphi\right) d \varphi .
$$

The component of gravity of the selected load section between the roller supports of the tubular conveyor, inclined at an angle $\alpha$ to the horizon, is determined by the formula:

$$
F_{g}=G \sin \alpha=\gamma S_{m} l_{p} \sin \alpha,
$$

where $G$ is the gravity of the selected portion of the load between the roller supports, N; $S_{m}$ is sectional area of a tubular belt filled with bulk load, $\mathrm{m}^{2}$.

According to Figure 2, the cross-sectional area of a tubular belt filled with bulk material is determined by the formula

$$
S_{m}=R^{2}\left[(\pi-2 \theta)+\frac{\sin 4 \theta}{2}\right]
$$

Substituting (21) and (22) into (1) taking into account (23), after integration, we obtain the equation for the limiting angle of inclination of the tubular conveyor $\alpha_{n}$ :

$$
d_{1} \cdot \operatorname{tg} \alpha_{n}=a_{1}+b_{1}\left(1+f^{2}+f \sqrt{f^{2}-\operatorname{tg}^{2} \alpha_{n}}\right)
$$


where $a_{1}=f_{1}\left[(\pi-2 \theta) \cos 2 \theta-\frac{\sin 4 \theta}{2} \cos 2 \theta+2 \sin 2 \theta-\frac{2 \sin ^{3} 2 \theta}{3}\right]$;

$$
b_{1}=f_{1}\left[(\pi-2 \theta) \cos 2 \theta+\frac{\sin 4 \theta}{2} \cos 2 \theta+\frac{2 \sin ^{3} 2 \theta}{3}\right] ; \quad d_{1}=(\pi-2 \theta)+\frac{\sin 4 \theta}{2} \text {. }
$$

Substituting into equation (24) $t=\operatorname{tg} \alpha_{n}$, we obtain the transcendental equation for $t$ :

$$
t=\frac{a_{1}+b_{1}\left(1+f^{2}+f \sqrt{f^{2}-t^{2}}\right)}{d_{1}} .
$$

From the right side of equation (25) it follows that $t \leq f$, i.e. $\alpha_{n} \leq \operatorname{arctg} f$.

After conversion, equation (25) can be represented as

$$
d_{1} t-a_{1}-b_{1}\left(1+f^{2}\right)=b_{1} f \sqrt{f^{2}-t^{2}} .
$$

Having squared the left and right sides of equality (26), after the transformation we obtain the quadratic equation for $t$ in the form:

$$
A_{1} t^{2}+B_{1} t+C_{1}=0
$$

where $A_{1}=d_{1}^{2}+b_{1}^{2} f^{2} ; \quad B_{1}=-2 d_{1}\left[a_{1}+b_{1}\left(1+f^{2}\right)\right] ; \quad C_{1}=\left[a_{1}+b_{1}\left(1+f^{2}\right)\right]^{2}-b_{1}^{2} f^{4}$.

Solving the quadratic equation (27) and taking a larger root, we obtain

$$
t=\frac{-B_{1}+\sqrt{B_{1}^{2}-4 A_{1} C_{1}}}{2 A_{1}}
$$

It follows from (28) that the positive roots of equation (27) exist if the condition $B_{1}^{2}-4 A C_{1} \geq 0$ is satisfied.

Finally, the maximum angle of inclination of the tubular belt conveyor $\alpha_{n}$ is determined from the equality

$$
\alpha_{n}=\operatorname{arctg} t
$$

\section{Results and discussion}

Studies have shown that at $\theta \rightarrow \pi / 2\left(90^{\circ}\right)$, i.e. with a small amount of bulk load in the tubular conveyor belt, the maximum angle of inclination of the conveyor $\alpha_{n} \rightarrow \operatorname{arctg} f_{1}$; moreover, $\alpha_{n} \geq \operatorname{arctg} f_{1}$ and, according to $(25), \alpha_{n} \leq \operatorname{arctg} f$.

Consequently, the limiting angle of inclination of the tubular conveyor in the case $f>f_{1}$ does not depend on the radius of the conveyor belt, is greater than or equal to the angle of friction of the load on the belt and less than or equal to the angle of internal friction of the bulk load, i.e. varies within

$$
\operatorname{arctg} f_{1} \leq \alpha_{n} \leq \operatorname{arctg} f .
$$

In Figures 3 and 4 show graphs of the dependence of the limiting angle of inclination of the tubular belt conveyor on the angle of unfilling of the conveyor belt $\theta$ for various values 
of the coefficient of friction of the load on the belt $f_{1}$. Moreover, the values of the coefficient of internal friction and the coefficients of friction of the load on the belt for ore took the values: $f=1 ; f_{1}=0.5 ; 0.6 ; 0.7 ; 0.8$ (Fig. 3 ), and for coal $f=0.58 ; f_{1}=0.3 ; 0.4 ; 0.5$ (Fig. 4).

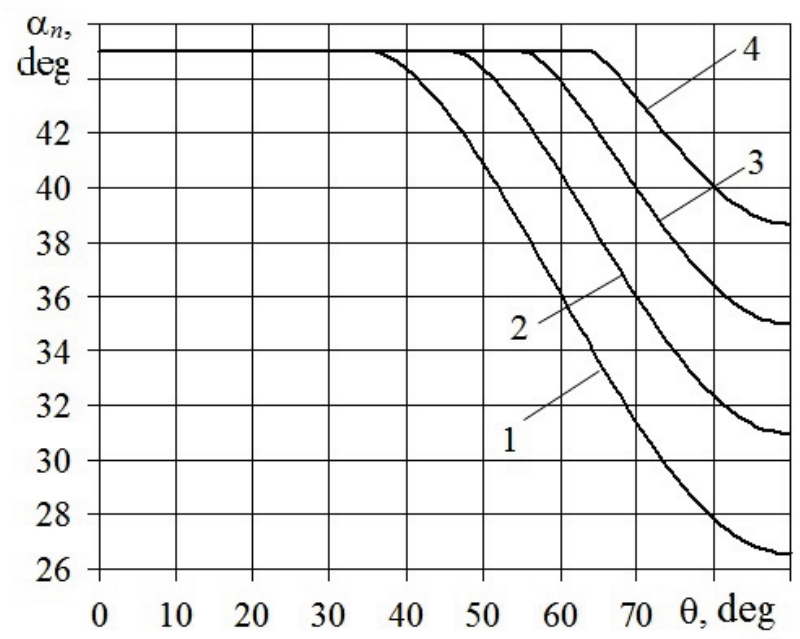

Fig. 3. A graph of the limit angle of inclination of the tubular belt conveyor $\alpha_{n}$ on the angle of unfilling of the conveyor belt $\theta$ for various values of the coefficient of friction of the load on the belt $f_{1}$ in the case of ore transportation: $1-f_{1}=0.5 ; 2-f_{1}=0.6 ; 3-f_{1}=0.7 ; 4-f_{1}=0.8$.

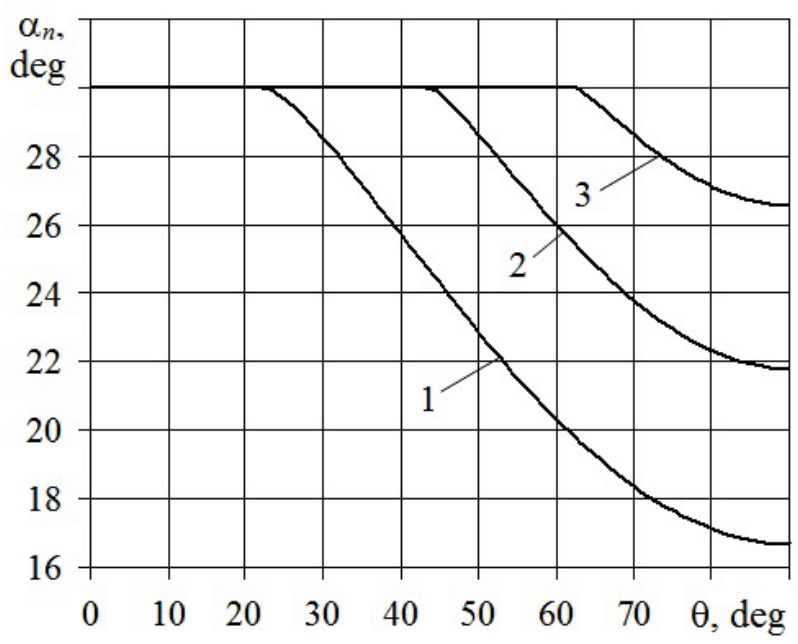

Fig. 4. Graph of the limit angle of inclination of the tubular belt conveyor $\alpha_{n}$ on the angle of unfilling of the conveyor belt $\theta$ for various values of the coefficient of friction of the load on the belt $f_{1}$ in the case of coal transportation: $1-f_{1}=0.3 ; 2-f_{1}=0.4 ; 3-f_{1}=0.5$.

Figures 3 and 4 show that with an increase in the angle of unfilling of the belt $\theta$ from 0 to the minimum value $\theta_{m}\left(0 \leq \theta \leq \theta_{m}\right)$, the limiting angle of inclination of the belt tubular conveyor assumes the maximum value equal to the angle of internal friction of the load, i.e. $\alpha_{n}=\operatorname{arctg} f$. And at values $\theta>\theta_{m}$ with increasing $\theta$, the limiting angle of inclination of the belt tubular conveyor decreases and at $\theta=\pi / 2\left(90^{\circ}\right)$ it takes a value equal to the angle of friction of the load on the conveyor belt, i.e. $\alpha_{n}=\operatorname{arctg} f_{1}$.

Moreover, for ore, the limiting angle of inclination of the tubular conveyor for the same 
values of the angle of unfilling $\theta$ is greater than for coal, and the maximum limiting angles of inclination of the tubular conveyor for ore and coal are $0,79\left(45^{\circ}\right)$ and $0,52\left(30^{\circ}\right)$, respectively.

In addition, from Figures 3 and 4 we have that for ore at $f_{1}=0.5 ; 0.6 ; 0.7 ; 0.8$, the minimum angle of unfilling the belt with a load $\theta_{m}$, respectively, is equal to: $\theta_{m}=0.63$ $\left(36^{\circ}\right) ; 0.82\left(47^{\circ}\right) ; 0.96\left(55^{\circ}\right) ; 1.12\left(64^{\circ}\right)$, and for coal at $f_{1}=0.3 ; 0.4 ; 0.5$, respectively, $\theta_{m}=0.40\left(23^{\circ}\right) ; 0.77\left(44^{\circ}\right) ; 1.1\left(63^{\circ}\right)$.

\section{Conclusions}

1. Based on the theory of the limiting equilibrium state of a granular medium, an analytical dependence of the limiting angle of inclination of a tubular belt conveyor on the degree of filling of the belt with the load and the properties of the bulk load is obtained.

2. At the same time, it was found that the maximum angle of inclination of the tubular belt conveyor depends on the angle of internal friction of the transported load, the angle of friction of the load on the conveyor belt, the degree of unfilling of the belt with load, and does not depend on the radius of the conveyor belt.

3. An analysis of this dependence showed that with an increase the degree of unfilling of the belt with load from 0 to the minimum value $\theta_{m}$, the limiting angle of inclination of the belt tube conveyor assumes the maximum value equal to the angle of internal friction of the load. And at $\theta>\theta_{m}$ with increasing $\theta$, the limiting angle of inclination of the belt tubular conveyor decreases and at $\theta=\pi / 2\left(90^{\circ}\right)$ it takes a value equal to the angle of friction of the load on the conveyor belt.

\section{References}

1. Perten, Yu. A. (1977). Krutonaklonnye konveyery. Leningrad: Mashinostroenie

2. Chernenko, V. D. (1985). Teoriya i raschet krutonaklonnykh konveyerov. Leningrad: Izdatelstvo LGU

3. Galkin, V. I., Dmitriev, V. G., Dyachenko, V. P. (2005). Sovremennaya teoriya lentochnykh konveyerov gornykh predpriyatiy. Moskva: MGGU

4. Kiriya, R. V., Larionov, M.G. (2015). Opredelenie predelnogo ugla naklona konveyera s lentoy glubokoy zhelobchatosti. Geo-Technical Mechanics, 120, 276-286

5. O. Voloshyn, O. Riabtsev. Some important aspects of rock mechanics and geomechanics. E3S Web of Conferences, International Conference Essays of Mining Science and Practice, 109 (2019). https://doi.org/10.1051/e3sconf/201910900114

6. Zenkov, R.L. (1964). Mechanika nasypnych gruzov. Moskva: Mashinostroenie 